\title{
SPACE GROUPS P1 AND Cc: HOW ARE THEY DOING?
}

Richard E. Marsh

The Beckman Institute

California Institute of Technology

Pasadena, Cal. 91125, USA 
TABLE 1S. Space group revisions; original space group, Cc (No. 9)

\begin{tabular}{|c|c|c|c|}
\hline Refcode & S. G. & Formula Unit & Reference \\
\hline AXIYUB & $\mathrm{C} 2 / \mathrm{c}$ & $\mathrm{C}_{32} \mathrm{H}_{36} \mathrm{O}_{6} \mathrm{Sn}$ & Ma et al. (2004a) \\
\hline AZERUS & $\mathrm{C} 2 / \mathrm{c}$ & $\mathrm{C}_{16} \mathrm{H}_{14} \mathrm{Cr}_{2} \mathrm{O}_{4} \mathrm{Se}$ & Song et al. (2004) \\
\hline BENWIB & Fdd2 & $\mathrm{C}_{54} \mathrm{H}_{68} \mathrm{~N}_{8} \mathrm{Ni}_{3} \mathrm{O}_{8} \cdot 2\left(\mathrm{PF}_{6}\right)$ & Adams et al. (2004) \\
\hline BIJKIP & P31c & $\mathrm{C}_{24} \mathrm{H}_{30} \mathrm{~N}_{3} \mathrm{P}$ & Beddie et al. (2004) \\
\hline BIJMIR & $\mathrm{C} 2 / \mathrm{c}$ & $\mathrm{C}_{44} \mathrm{H}_{70} \mathrm{O}_{2} \mathrm{Si}_{4} \mathrm{Zr}$ & Bradley et al. (2004) \\
\hline DADMEB & $\mathrm{C} 2 / \mathrm{c}$ & $\mathrm{C}_{4} \mathrm{H}_{10} \mathrm{~N}_{2} \mathrm{O}_{10} \mathrm{Zn}$ & Yang et al. (2004) \\
\hline DALNOU & $\mathrm{C} 2 / \mathrm{c}$ & $\mathrm{C}_{33} \mathrm{H}_{52} \mathrm{ClN}_{7} \mathrm{ORu} \cdot \mathrm{CF}_{3} \mathrm{SO}_{3}$ & Jitsukawa et al. (2004) \\
\hline EBUXUV & $\mathrm{C} 2 / \mathrm{c}$ & $\mathrm{C}_{42} \mathrm{H}_{40} \mathrm{~N}_{2} \mathrm{O}_{2}$ & Shen et al. (2004) \\
\hline EVUQUH & $\mathrm{C} 2 / \mathrm{c}$ & $\mathrm{C}_{34} \mathrm{H}_{55} \mathrm{~N}_{6} \mathrm{Rh} \cdot 2\left(\mathrm{PF}_{6}\right)$ & Mas-Marza et al. (2004) \\
\hline EXATUS & Fdd2 & $\mathrm{C}_{68} \mathrm{H}_{54} \mathrm{Co}_{4} \mathrm{O}_{8} \mathrm{P}_{4}$ & Golovko et al. (2004) \\
\hline FAYQUS & $\mathrm{C} 2 / \mathrm{c}$ & $\mathrm{C}_{14} \mathrm{H}_{14} \mathrm{~N}_{2} \mathrm{O}_{4} \mathrm{~S}_{2} \mathrm{Zn}$ & Ng et al. (2004) \\
\hline FEJGAD & $\mathrm{C} 2 / \mathrm{c}$ & $\mathrm{C}_{24} \mathrm{H}_{22} \mathrm{Cl}_{2} \mathrm{~N}_{2} \mathrm{Sn}$ & Ma et al. (2004b) \\
\hline IBUSUU & Ama2 & $\mathrm{C}_{26} \mathrm{H}_{26} \mathrm{GaNO}_{2} \mathrm{P}_{2}$ & Montiel-Palma et al. (2004) \\
\hline IREFEQ & $\mathrm{C} 2 / \mathrm{c}$ & $\mathrm{C}_{54} \mathrm{H}_{18} \mathrm{~F}_{20} \mathrm{~N}_{6} \mathrm{Ru} \cdot 3\left(\mathrm{CH}_{2} \mathrm{Cl}_{2}\right)$ & Li et al. (2004) \\
\hline IXUVOM & $\mathrm{C} 2 / \mathrm{c}$ & $\mathrm{C}_{18} \mathrm{H}_{15} \mathrm{NO}_{4}$ & Costa et al. (2004) \\
\hline SAGJIU & $\mathrm{C} 2 / \mathrm{c}$ & $\mathrm{C}_{23} \mathrm{H}_{27} \mathrm{BF}_{2} \mathrm{O}_{4}$ & Mayoral et al. (2004) \\
\hline SALTUV & $\mathrm{C} 2 / \mathrm{c}$ & $\mathrm{C}_{10} \mathrm{H}_{16} \mathrm{~N}_{10} \mathrm{~S}_{2} \mathrm{Zn}$ & $\mathrm{Du}(2004)$ \\
\hline WAGRAY & $\mathrm{C} 2 / \mathrm{c}$ & $\mathrm{C}_{41} \mathrm{H}_{45} \mathrm{ClN}_{4} \mathrm{O}_{4} \cdot \mathrm{C}_{10} \mathrm{H}_{9} \mathrm{~N}_{3} \mathrm{O}_{7}$ & Oliva et al. (2004) \\
\hline WAJDIV & $\mathrm{C} 2 / \mathrm{c}$ & $\mathrm{C}_{35} \mathrm{H}_{30} \mathrm{FeN}_{12} \mathrm{O}_{3} \mathrm{Sr}$ & RoyChowdhury et al. (2004) \\
\hline XACMOE & $\mathrm{C} 2 / \mathrm{c}$ & $\mathrm{C}_{22} \mathrm{H}_{28} \mathrm{~N}_{4} \mathrm{O}_{4} \mathrm{Zn}$ & Chantarasiri et al. (2004) \\
\hline YACPAU & $\mathrm{C} 2 / \mathrm{c}$ & $\mathrm{C}_{38} \mathrm{H}_{36} \mathrm{FeN}_{6} \cdot 2\left(\mathrm{PF}_{6}\right)$ & Brady et al. (2004) \\
\hline YAPZAR & $\mathrm{C} 2 / \mathrm{c}$ & $\mathrm{C}_{90} \mathrm{H}_{172} \mathrm{Li}_{4} \mathrm{NO}_{24} \mathrm{Si}_{16} \mathrm{Yb}$ & Lorenz et al. (2004) \\
\hline CEBLUR & $\mathrm{C} 2 / \mathrm{c}$ & $\mathrm{C}_{12} \mathrm{H}_{8} \mathrm{~N}_{4} \mathrm{~S}_{4} \mathrm{Zn}$ & Suen et al. (2005) \\
\hline CEFDOH & $\mathrm{C} 2 / \mathrm{c}$ & $\mathrm{C}_{104} \mathrm{H}_{28} \mathrm{~F}_{40} \mathrm{~N}_{10} \cdot 3\left(\mathrm{C}_{7} \mathrm{H}_{16}\right) \cdot 1.5\left(\mathrm{H}_{2} \mathrm{O}\right) \cdot 2\left(\mathrm{CH}_{2} \mathrm{Cl}_{2}\right)$ & Anand et al. (2005) \\
\hline ECATIM & $\mathrm{C} 2 / \mathrm{c}$ & $\mathrm{C}_{12} \mathrm{H}_{12} \mathrm{O}_{15} \mathrm{~Tb} \cdot \mathrm{Na}_{3} \cdot 8\left(\mathrm{H}_{2} \mathrm{O}\right)$ & Kang et al. (2005) \\
\hline FENWUR & $\mathrm{C} 2 / \mathrm{c}$ & $\mathrm{C}_{52} \mathrm{H}_{52} \mathrm{ZnN}_{16} \mathrm{O}_{2} \cdot 2\left(\mathrm{NO}_{3}\right) \cdot 5\left(\mathrm{H}_{2} \mathrm{O}\right)$ & Zhu et al. (2005) \\
\hline FIPGOB & $\mathrm{C} 2 / \mathrm{c}$ & $\mathrm{C}_{38} \mathrm{H}_{24} \mathrm{CdN}_{4} \mathrm{O}_{4} \cdot 2\left(\mathrm{H}_{2} \mathrm{O}\right)$ & Shi et al. (2005) \\
\hline JARNOG & $\mathrm{C} 2 / \mathrm{c}$ & $\mathrm{C}_{26} \mathrm{H}_{28} \mathrm{CdN}_{10} \mathrm{O}_{6} \cdot \mathrm{CH}_{2} \mathrm{Cl}_{2}$ & Yeh et al. (2005) \\
\hline LAMDIN & Fdd2 & $\mathrm{C}_{12} \mathrm{H}_{36} \mathrm{CaO}_{6} \mathrm{~S}_{6} \cdot 2\left(\mathrm{ClO}_{4}\right)$ & Ullstrom et al. (2005) \\
\hline LARTII & $\mathrm{C} 2 / \mathrm{c}$ & $\mathrm{C}_{19} \mathrm{H}_{30} \mathrm{~N}_{3} \mathrm{O}_{2} \mathrm{P}$ & Gholivand et al. (2005) \\
\hline LEBWAR & Fdd2 & $\mathrm{C}_{11} \mathrm{H}_{16} \mathrm{NS} \cdot \mathrm{C}_{2} \mathrm{HO}_{4}$ & Ananikov et al. (2005) \\
\hline NATLAW & $\mathrm{C} 2 / \mathrm{c}$ & $\mathrm{C}_{27} \mathrm{H}_{36} \mathrm{AuClN}{ }_{2} \cdot \mathrm{C}_{7} \mathrm{H}_{8}$ & Fructos et al. (2005) \\
\hline NAVTUA & $\mathrm{C} 2 / \mathrm{c}$ & $\mathrm{C}_{20} \mathrm{H}_{28} \mathrm{AuN}_{8} \mathrm{O}_{8} \cdot \mathrm{Na}_{3} \cdot 2\left(\mathrm{C}_{5} \mathrm{H}_{7} \mathrm{~N}_{2} \mathrm{O}_{2}\right) \cdot 8\left(\mathrm{H}_{2} \mathrm{O}\right)$ & Oyaizu et al. (2005) \\
\hline NICJEP & $\mathrm{C} 2 / \mathrm{c}$ & $\mathrm{C}_{13} \mathrm{H}_{12} \mathrm{O}_{3}$ & Wang et al. (2005) \\
\hline TASDUN & Fdd2 & $\mathrm{C}_{21} \mathrm{H}_{18} \mathrm{O}_{3}$ & Muthusamy et al. (2005) \\
\hline TAVDAW & $\mathrm{C} 2 / \mathrm{c}$ & $\mathrm{C}_{25} \mathrm{H}_{45} \mathrm{~N}_{3} \mathrm{O}_{5}$ & Hanessian et al. (2005) \\
\hline TAWFIH & $\mathrm{C} 2 / \mathrm{c}$ & $\mathrm{C}_{24} \mathrm{H}_{18} \mathrm{FeN}_{12}$ & Gaspar et al. (2005) \\
\hline WARTEP & $\mathrm{C} 2 / \mathrm{c}$ & $\mathrm{C}_{36} \mathrm{H}_{24} \mathrm{~N}_{6} \mathrm{Ni} \cdot 2\left(\mathrm{C}_{9} \mathrm{H}_{12} \mathrm{NO}_{5} \mathrm{~S}_{2}\right) \cdot \mathrm{H}_{2} \mathrm{O}$ & Lan et al. (2005) \\
\hline YAJXIR & $\mathrm{C} 2 / \mathrm{c}$ & $\mathrm{C}_{21} \mathrm{H}_{24} \mathrm{~N}_{3} \mathrm{O}_{2} \cdot \mathrm{C}_{6} \mathrm{H}_{2} \mathrm{~N}_{3} \mathrm{O}_{7}$ & Voskressensky et al. (2005) \\
\hline DEJCOL & $\mathrm{C} 2 / \mathrm{c}$ & $\mathrm{C}_{43} \mathrm{H}_{49} \mathrm{~N}_{5} \cdot \mathrm{CH}_{2} \mathrm{Cl}_{2}$ & Champouret et al. (2006) \\
\hline DELJAG & $\mathrm{C} 2 / \mathrm{c}$ & $\mathrm{C}_{26} \mathrm{H}_{24} \mathrm{FeN}_{6} \mathrm{Se}_{2}$ & Morita et al. (2006) \\
\hline DIFBUQ & $\mathrm{C} 2 / \mathrm{c}$ & $\mathrm{C}_{32} \mathrm{H}_{40} \mathrm{~F}_{12} \mathrm{HfO}_{8}$ & Zherikova et al. (2006) \\
\hline GEFYEW & $\mathrm{C} 2 / \mathrm{c}$ & $\mathrm{C}_{42} \mathrm{H}_{46} \mathrm{Co}_{2} \mathrm{~N}_{14} \mathrm{O}_{12} \cdot 2\left(\mathrm{H}_{2} \mathrm{O}\right)$ & Fernandez-Fernandez et al. (2006a) \\
\hline HEBZUK & $\mathrm{C} 2 / \mathrm{c}$ & $\mathrm{C}_{24} \mathrm{H}_{32} \mathrm{~B}_{2} \mathrm{BiN}_{12} \mathrm{~S}_{6} \cdot \mathrm{NO}_{2}(?) \cdot 2.5\left(\mathrm{C}_{3} \mathrm{H}_{7} \mathrm{NO}\right)$ & Dodds et al. (2006) \\
\hline KIBFUX & $\mathrm{C} 2 / \mathrm{c}$ & $\mathrm{C}_{6} \mathrm{H}_{24} \mathrm{MnN}_{6} \cdot \mathrm{C}_{10} \mathrm{H}_{6} \mathrm{~S}_{2} \cdot \mathrm{H}_{2} \mathrm{O}$ & Yu et al. (2006) \\
\hline KIBGAE & $\mathrm{C} 2 / \mathrm{c}$ & $\mathrm{C}_{6} \mathrm{H}_{24} \mathrm{CoN}_{6} \cdot \mathrm{C}_{10} \mathrm{H}_{6} \mathrm{~S}_{2} \cdot \mathrm{H}_{2} \mathrm{O}$ & Yu et al. (2006) \\
\hline KIBGEI & $\mathrm{C} 2 / \mathrm{c}$ & $\mathrm{C}_{6} \mathrm{H}_{24} \mathrm{NiN}_{6} \cdot \mathrm{C}_{10} \mathrm{H}_{6} \mathrm{~S}_{2} \cdot \mathrm{H}_{2} \mathrm{O}$ & Yu et al. (2006) \\
\hline NEJTOM & $\mathrm{C} 2 / \mathrm{c}$ & $\mathrm{C}_{58} \mathrm{H}_{58} \mathrm{CuN}_{10} \mathrm{O}_{4} \cdot 2\left(\mathrm{ClO}_{4}\right) \cdot 0.5\left(\mathrm{C}_{6} \mathrm{H}_{6}\right) \cdot 2.5\left(\mathrm{C}_{2} \mathrm{H}_{3} \mathrm{~N}\right.$ & J)Plante \& Glass (2006) \\
\hline NEKFEP & Fdd2 & $\mathrm{C}_{42} \mathrm{H}_{48}$ & Muller et al. (2006) \\
\hline NIMPIJ & $\mathrm{C} 2 / \mathrm{c}$ & $\mathrm{C}_{34} \mathrm{H}_{28} \mathrm{Cu}_{2} \mathrm{~N}_{4} \mathrm{O}_{8}$ & Tao et al. (2006) \\
\hline SERXOD & $\mathrm{C} 2 / \mathrm{c}$ & $\mathrm{C}_{86} \mathrm{H}_{110} \mathrm{~N}_{4} \mathrm{O}_{22} \cdot 2\left(\mathrm{CH}_{4} \mathrm{O}\right)$ & Zhang et al. (2006) \\
\hline XEFCUH & $\mathrm{C} 2 / \mathrm{c}$ & $\mathrm{C}_{30} \mathrm{H}_{38} \mathrm{Ag}_{2} \mathrm{~N}_{10} \cdot 2\left(\mathrm{ClO}_{4}\right) \cdot 2\left(\mathrm{C}_{2} \mathrm{H}_{3} \mathrm{~N}\right)$ & Fernandez-Fernandez et al. (2006b) \\
\hline
\end{tabular}


TABLE 1S. Space group revisions; original space group, Cc (No. 9) - Continued

$\begin{array}{lll}\text { Refcode } & \text { S. G. } & \text { Formula Unit } \\ \text { CIBJED } & \mathrm{C} 2 / \mathrm{c} & \mathrm{C}_{22} \mathrm{H}_{34} \mathrm{~N}_{2} \mathrm{O}_{4} \mathrm{Ti} \\ \text { HIWMAC } & \mathrm{C} 2 / \mathrm{c} & 2\left(\mathrm{C}_{12} \mathrm{H}_{28} \mathrm{~N}\right) \cdot \mathrm{C}_{35} \mathrm{H}_{65} \mathrm{Co}_{2} \mathrm{~N}_{13} \mathrm{O}_{5} \cdot \mathrm{C}_{4} \mathrm{H}_{9} \mathrm{NO} \\ \text { KIJCOW } & \mathrm{C} 2 / \mathrm{c} & \mathrm{C}_{30} \mathrm{H}_{20} \mathrm{MoN}_{2} \mathrm{O}_{4} \\ \text { SICNAU } & \mathrm{C} 2 / \mathrm{c} & 2\left(\mathrm{C}_{40} \mathrm{H}_{38} \mathrm{~N}_{4} \mathrm{O}_{8}\right) \cdot 3\left(\mathrm{CHCl}_{3}\right) \cdot 6\left(\mathrm{H}_{2} \mathrm{O}\right) \\ \text { TICZAH } & \mathrm{C} 2 / \mathrm{c} & \mathrm{C}_{24} \mathrm{H}_{52} \mathrm{O}_{2} \mathrm{Si}_{2} \\ \text { TIZZIM } & \mathrm{C} 2 / \mathrm{c} & \mathrm{C}_{7} \mathrm{H}_{14} \mathrm{~N}_{2} \\ \text { YIDGAU } & \mathrm{C} 2 / \mathrm{c} & \mathrm{C}_{44} \mathrm{H}_{34} \mathrm{IrN}_{6} \cdot 3\left(\mathrm{PF}_{6}\right) \cdot 2\left(\mathrm{C}_{2} \mathrm{H}_{3} \mathrm{~N}\right) \\ & & \\ \text { BOJWUT } & \mathrm{C} 2 / \mathrm{c} & \mathrm{C}_{10} \mathrm{H}_{18} \mathrm{CdN}_{4} \mathrm{O}_{4} \mathrm{~S}_{2} \\ \text { CIRZUZ } & \mathrm{C} 2 / \mathrm{c} & \mathrm{C}_{54} \mathrm{H}_{76} \mathrm{CuN}_{4} \cdot \mathrm{PF}_{6} \\ \text { DELWEX01 } & \mathrm{C} 2 / \mathrm{c} & \mathrm{C}_{23} \mathrm{H}_{24} \mathrm{CuN}_{2} \mathrm{O}_{2} \\ \text { JIYGAA } & \mathrm{C} 2 / \mathrm{c} & \mathrm{C}_{22} \mathrm{H}_{22} \mathrm{~N}_{4} \mathrm{~S} \\ \text { NIYXID } & \mathrm{C} 2 / \mathrm{c} & \mathrm{Na}_{16} \mathrm{C}_{16} \mathrm{H}_{24} \mathrm{GaN}_{4} \mathrm{O}_{8} \cdot 5\left(\mathrm{H}_{2} \mathrm{O}\right) \\ \text { QOBJAT } & \mathrm{C} 2 / \mathrm{c} & \mathrm{C}_{44} \mathrm{H}_{32} \mathrm{~N}_{8} \mathrm{Ni}_{2} \mathrm{O}_{12} \cdot 2\left(\mathrm{H}_{2} \mathrm{O}\right) \\ \text { RIWNER } & \mathrm{C} 2 / \mathrm{c} & \mathrm{C}_{31} \mathrm{H}_{57} \mathrm{FeN}_{2} \mathrm{O}_{2} \mathrm{ScSi}_{2} \\ \text { VIZHIW01 } & \mathrm{C} 2 / \mathrm{c} & \mathrm{C}_{16} \mathrm{H}_{22} \mathrm{O}_{4}\end{array}$

Reference

Boyle et al. (2007)

Zinn et al. (2007)

Sakakura et al. (2007)

Chawla et al. (2007)

Yamada et al. (2007)

Morozova et al. (2007)

Yoshikawa et al. (2007)

Zhu et al. (2008)

Diez-Gonzales et al. (2008)

Sarkar et al. (2008)

Zheng et al. (2008)

Heppeler et al. (2008)

Wang et al. (2008)

Carver et al. (2008)

Liskin \& Valente (2008)

Revisions to space groups other than P1 or Cc

\begin{tabular}{|c|c|c|c|c|}
\hline Refcode & Old & New & Formula Unit & Reference \\
\hline BENWOH & $\mathrm{P} 2_{1}$ & $\mathrm{C} 222_{1}$ & $\mathrm{C}_{62} \mathrm{H}_{80} \mathrm{~N}_{8} \mathrm{NaNi}_{4} \mathrm{O}_{16} \cdot \mathrm{ClO}_{4}$ & Adams et al. (2004) \\
\hline BENWUN & $\mathrm{C} 2$ & $\mathrm{C} 222_{1}$ & $\mathrm{C}_{62} \mathrm{H}_{80} \mathrm{~N}_{8} \mathrm{NaNi}_{4} \mathrm{O}_{16} \cdot \mathrm{PF}_{6}$ & Adams et al. (2004) \\
\hline DALNAG & $\mathrm{P} 2_{1}$ & $\mathrm{P} 2{ }_{1} / \mathrm{m}$ & $\mathrm{C}_{28} \mathrm{H}_{40} \mathrm{Cl}_{2} \mathrm{~N}_{6} \mathrm{Ru} \cdot \mathrm{PF}_{6} \cdot 2\left(\mathrm{H}_{2} \mathrm{O}\right)$ & Jitsukawa et al. (2004) \\
\hline FENXOM & $\mathrm{C} 2$ & $\mathrm{C} 2 / \mathrm{c}$ & $\mathrm{C}_{26} \mathrm{H}_{24} \mathrm{CuN}_{8} \mathrm{O}_{4} \mathrm{~S} \cdot 8.5\left(\mathrm{H}_{2} \mathrm{O}\right)$ & Zhu et al. (2005) \\
\hline FENXUS & $\mathrm{P} \overline{1}$ & $\mathrm{C} 2 / \mathrm{c}$ & $\mathrm{C}_{26} \mathrm{H}_{24} \mathrm{CoN}_{8} \mathrm{O}_{4} \mathrm{~S} \cdot 8.25\left(\mathrm{H}_{2} \mathrm{O}\right)$ & Zhu et al. (2005) \\
\hline LARTOO & $\mathrm{P} \overline{1}$ & $\mathrm{C} 2 / \mathrm{c}$ & $\mathrm{C}_{17} \mathrm{H}_{26} \mathrm{~N}_{3} \mathrm{O}_{2} \mathrm{P}$ & Gholivand et al. (2005) \\
\hline DELJEK & $\mathrm{P} 22_{1} / \mathrm{a}$ & Pbam & $\mathrm{C}_{26} \mathrm{H}_{24} \mathrm{FeN}_{6} \mathrm{Se}_{2} \cdot 2\left(\mathrm{C}_{2} \mathrm{H}_{6} \mathrm{O}\right)$ & Morita et al. (2006) \\
\hline DIFGIJ & $\mathrm{P} \overline{1}$ & $\mathrm{P} 22_{1} / \mathrm{c}$ & $\mathrm{C}_{10} \mathrm{H}_{8} \mathrm{~F}_{6} \mathrm{O}_{4} \mathrm{Pt}$ & Zharkova et al. (2006). \\
\hline NIMPOP & $\mathrm{Pc}$ & $\mathrm{P} 21_{1} / \mathrm{c}$ & $\mathrm{C}_{35} \mathrm{H}_{32} \mathrm{Cu}_{2} \mathrm{MgN}_{4} \mathrm{O}_{10} \cdot \mathrm{CH}_{4} \mathrm{O}$ & Tao et al. (2006) \\
\hline CIBKAA & $\mathrm{P} \overline{1}$ & $\mathrm{C} 2 / \mathrm{c}$ & $\mathrm{C}_{44} \mathrm{H}_{50} \mathrm{~N}_{2} \mathrm{O}_{4} \mathrm{Ti}$ & Boyle et al. (2007) \\
\hline TIZZAE & Pnc2 & Pbcn & $\mathrm{C}_{14} \mathrm{H}_{26} \mathrm{~N}_{4} \mathrm{Cu}$ & Morozova et al. (2007) \\
\hline
\end{tabular}


TABLE 2S. Space group revisions; original space group, P1 (No. 1)

\begin{tabular}{|c|c|c|c|}
\hline Refcode & S. G. & Formula Unit & Reference \\
\hline CEGJUU & $\mathrm{Cc}$ & $\mathrm{C}_{14} \mathrm{H}_{23} \mathrm{NO}_{7} \mathrm{Sn}_{2} \cdot \mathrm{C}_{12} \mathrm{H}_{8} \mathrm{~N}_{2}$ & Ma et al. (2006) \\
\hline CEPKOY & $\mathrm{Cc}$ & $\mathrm{C}_{22} \mathrm{H}_{14} \mathrm{O}_{3}$ & Zhu et al. (2006) \\
\hline DELHUY & $\mathrm{P} \overline{1}$ & $\mathrm{C}_{26} \mathrm{H}_{24} \mathrm{FeN}_{6} \mathrm{~S}_{2} \cdot \mathrm{C}_{2} \mathrm{H}_{6} \mathrm{O}$ & Morita et al. (2006) \\
\hline DERMET & $\mathrm{P} \overline{1}$ & $\mathrm{C}_{38} \mathrm{H}_{46} \mathrm{~N}_{2} \mathrm{NiS}$ & Malyshev et al. (2006) \\
\hline DESNUL & $\mathrm{P} \overline{1}$ & $\mathrm{C}_{24} \mathrm{H}_{44} \mathrm{O}_{2}$ & Wang et al. (2006) \\
\hline DIDTIU & $\mathrm{P} \overline{1}$ & $\mathrm{C}_{10} \mathrm{H}_{8} \mathrm{~F}_{6} \mathrm{O}_{4} \mathrm{Pt}$ & Zharkova et al. (2006) \\
\hline ECEHAW & $\mathrm{P} 2_{1}$ & $\mathrm{C}_{26} \mathrm{H}_{16} \mathrm{~N}_{4} \mathrm{OS}$ & Ogura et al. (2006) \\
\hline HEQZAF & $\mathrm{P} \overline{1}$ & $2\left(\mathrm{C}_{10} \mathrm{H}_{21} \mathrm{~N}_{2}\right) \cdot \mathrm{C}_{6} \mathrm{NiS}_{10}$ & Onozaki \& Miyamura (2006) \\
\hline JECROZ & $\mathrm{P} 2_{1}$ & $\mathrm{C}_{36} \mathrm{H}_{48} \mathrm{Cl}_{4} \mathrm{Cu}_{2} \mathrm{~N}_{4} \mathrm{O}_{4}$ & Hatano et al. (2006) \\
\hline KEVKIG & $\mathrm{P} \overline{1}$ & $\mathrm{C}_{14} \mathrm{H}_{16} \mathrm{Fe}_{2} \mathrm{O}_{9} \mathrm{~S}_{2}$ & Song et al. (2006) \\
\hline PEDSUN & $\mathrm{P} \overline{1}$ & $\mathrm{C}_{16} \mathrm{H}_{14} \mathrm{~N}_{6} \mathrm{Ni}_{2} \mathrm{O}_{2} \mathrm{~S}_{4}$ & Shi et al. (2006) \\
\hline SEWXUO & $\mathrm{P} \overline{4} \mathrm{n} 2$ & $\mathrm{C}_{32} \mathrm{H}_{30} \mathrm{Co}_{2} \mathrm{CrLaN}_{8} \mathrm{O}_{12}$ & Shiga et al. (2006) \\
\hline SEWYAV & $\mathrm{P} \overline{4} \mathrm{n} 2$ & $\mathrm{C}_{32} \mathrm{H}_{30} \mathrm{Co}_{2} \mathrm{CrGdN}_{8} \mathrm{O}_{12}$ & Shiga et al. (2006) \\
\hline TEJCAN & $\mathrm{P} 2_{1}$ & $\mathrm{C}_{40} \mathrm{H}_{40} \mathrm{FeN}_{2} \mathrm{PPd} \cdot \mathrm{SbF}_{6} \cdot \mathrm{CH}_{2} \mathrm{Cl}_{2}$ & Hintermann et al. (2006) \\
\hline UCUNOW & $\mathrm{P} \overline{1}$ & $\mathrm{C}_{24} \mathrm{H}_{20} \mathrm{As} \cdot \mathrm{C}_{6} \mathrm{H}_{12} \mathrm{Cl}_{8} \mathrm{~N}_{3} \mathrm{O}_{3} \mathrm{Pt}_{2} \cdot 0.5\left(\mathrm{C}_{5} \mathrm{H}_{12}\right)$ & Pellicani et al. (2006) \\
\hline VENPOU & $\mathrm{P} 2{ }_{1} 2_{1} 2_{1}$ & $\mathrm{C}_{23} \mathrm{H}_{14} \mathrm{~F}_{6} \mathrm{O}_{5} \mathrm{~S}_{2}$ & Hasegawa et al. (2006) \\
\hline VIMBID & $\mathrm{P} \overline{1}$ & $\mathrm{C}_{28} \mathrm{H}_{48} \mathrm{~N}_{2} \mathrm{O}_{4}$ & Uncuta et al. (2006) \\
\hline ZENVAP01 & $\mathrm{P} \overline{1}$ & $\mathrm{C}_{13} \mathrm{H}_{12} \mathrm{O}$ & Babkov et al. (2006) \\
\hline CIBLOP & $\mathrm{P} \overline{1}$ & $\mathrm{C}_{24} \mathrm{H}_{22} \mathrm{~N}_{2} \mathrm{O}_{4} \mathrm{~S}_{2} \mathrm{Ti}$ & Boyle et al. (2007) \\
\hline CIWFEU & $\mathrm{P} \overline{1}$ & $\mathrm{C}_{32} \mathrm{H}_{18} \mathrm{~F}_{4}$ & Ie et al. (2007) \\
\hline CIWGAR & $\mathrm{P} \overline{1}$ & $\mathrm{C}_{36} \mathrm{H}_{16} \mathrm{~F}_{10} \mathrm{O}_{2}$ & Ie et al. (2007) \\
\hline DICREN & $\mathrm{P} \overline{1}$ & $\mathrm{C}_{21} \mathrm{H}_{31} \mathrm{~N}_{5} \mathrm{O}_{6}$ & Comelles et al. (2007) \\
\hline DIHXUO & $\mathrm{P} \overline{1}$ & $2\left(\mathrm{C}_{12} \mathrm{H}_{16} \mathrm{~N}_{8} \mathrm{Zn}\right) \cdot \mathrm{C}_{6} \mathrm{H}_{8} \mathrm{Mo}_{8} \mathrm{~N}_{4} \mathrm{O}_{26} \cdot 6\left(\mathrm{H}_{2} \mathrm{O}\right)$ & Tian et al. (2007a) \\
\hline GIJQUM & $\mathrm{P} \overline{1}$ & $\mathrm{C}_{34} \mathrm{H}_{52} \mathrm{CoP} \cdot 2 \mathrm{PF}_{6}$ & Yu et al. (2007) \\
\hline HEYHEZ & $\mathrm{P} \overline{1}$ & $\mathrm{C}_{28} \mathrm{H}_{34} \mathrm{Cl}_{2} \mathrm{Cu}_{2} \mathrm{~N}_{6} \cdot 2\left(\mathrm{ClO}_{4}\right)$ & Liang et al. (2007) \\
\hline HEZJIG & $\mathrm{P} \overline{1}$ & $2\left(\mathrm{C}_{19} \mathrm{H}_{14} \mathrm{Cl}_{2} \mathrm{~N}_{2} \mathrm{O}_{2}\right) \cdot \mathrm{CH}_{2} \mathrm{Cl}_{2}$ & Cheng \& Cheng (2007) \\
\hline HIDJUA & $\mathrm{P} \overline{1}$ & $\mathrm{C}_{11} \mathrm{H}_{14} \mathrm{~N}_{2} \mathrm{O}_{2} \mathrm{~S}$ & Lin et al. (2007) \\
\hline HIGHAH & $\mathrm{P} \overline{1}$ & $\mathrm{C}_{19} \mathrm{H}_{14} \mathrm{O}_{2}$ & Kavala et al. (2007) \\
\hline KEYSAJ & $\mathrm{P} \overline{1}$ & $2\left(\mathrm{C}_{3} \mathrm{H}_{10} \mathrm{~N}\right) \cdot \mathrm{C}_{12} \mathrm{H}_{8} \mathrm{O}_{6} \mathrm{~S}_{2} \cdot \mathrm{C}_{4} \mathrm{H}_{8} \mathrm{O}_{2}$ & Mizobe et al. (2007) \\
\hline KEYSIR & $\mathrm{P} \overline{1}$ & $2\left(\mathrm{C}_{3} \mathrm{H}_{10} \mathrm{~N}\right) \cdot \mathrm{C}_{12} \mathrm{H}_{8} \mathrm{O}_{6} \mathrm{~S}_{2} \cdot \mathrm{C}_{4} \mathrm{H}_{8} \mathrm{O}_{2}$ & Mizobe et al. (2007) \\
\hline KICDEG & $\mathrm{P} \overline{1}$ & $\mathrm{C}_{18} \mathrm{H}_{24} \mathrm{CoN}_{12} \cdot 2\left(\mathrm{C}_{7} \mathrm{H}_{4} \mathrm{NO}_{4}\right) \cdot 2\left(\mathrm{H}_{2} \mathrm{O}\right)$ & Shi et al. (2007) \\
\hline KIDYOM & $\mathrm{P} \overline{1}$ & $\mathrm{C}_{32} \mathrm{H}_{36} \mathrm{Ag}_{2} \mathrm{~N}_{8} \mathrm{O}_{4} \cdot 2\left(\mathrm{ClO}_{4}\right) \cdot 2\left(\mathrm{C}_{2} \mathrm{H}_{3} \mathrm{~N}\right)$ & Chen et al. (2007) \\
\hline KIDZIH & $\mathrm{P} \overline{1}$ & $\mathrm{C}_{32} \mathrm{H}_{36} \mathrm{Ag}_{2} \mathrm{~N}_{8} \mathrm{O}_{4} \cdot 2\left(\mathrm{BF}_{4}\right) \cdot \mathrm{C}\left({ }_{2} \mathrm{H}_{3} \mathrm{~N}\right) \cdot \mathrm{H}_{2} \mathrm{O}$ & Chen et al. (2007) \\
\hline LEZLEI & $\mathrm{P} 2_{1}$ & $\mathrm{C}_{35} \mathrm{H}_{32} \mathrm{O}_{5}$ & Shimasaki et al. (2007) \\
\hline MILDUH & $\mathrm{P} 2_{1}$ & $\mathrm{C}_{18} \mathrm{H}_{28} \mathrm{C}_{12} \mathrm{Cl}_{2} \mathrm{~N}_{2} \mathrm{P}_{2} \mathrm{Pd} \cdot \mathrm{CH}_{2} \mathrm{Cl}_{2}$ & Imamoto et al. (2007) \\
\hline ODUWEQ & $\mathrm{P} \overline{1}$ & $\mathrm{C}_{6} \mathrm{H}_{8} \mathrm{~N} \cdot \mathrm{C}_{6} \mathrm{H}_{7} \mathrm{~N} \cdot \mathrm{H}_{4} \mathrm{~B}_{5} \mathrm{O}_{10}$ & Beckett et al. (2007) \\
\hline RIFSEF & Fdd2 & $\mathrm{C}_{12} \mathrm{H}_{24} \mathrm{Br}_{3} \mathrm{FeN}_{4} \mathrm{O}_{2}$ & Lundberg et al. (2007) \\
\hline TIDXAG & $\mathrm{P} \overline{1}$ & $\mathrm{C}_{62} \mathrm{H}_{40} \mathrm{Br}_{6} \mathrm{~N}_{4} \mathrm{O}_{12} \mathrm{~Tb}_{2} \cdot 2\left(\mathrm{C}_{7} \mathrm{H}_{5} \mathrm{BrO}_{2}\right) \cdot 2\left(\mathrm{H}_{2} \mathrm{O}\right)$ & Li et al. (2007a) \\
\hline TIDXEK & $\mathrm{P} \overline{1}$ & $\mathrm{C}_{52} \mathrm{H}_{38} \mathrm{Br}_{4} \mathrm{~N}_{4} \mathrm{O}_{12} \mathrm{~Tb}_{2}$ & Li et al. (2007a) \\
\hline TILDUO & $\mathrm{P} \overline{1}$ & $\mathrm{C}_{56} \mathrm{H}_{34} \mathrm{Eu}_{2} \mathrm{~N}_{4} \mathrm{O}_{20}$ & Tian et al. (2007b) \\
\hline TILROW & $\mathrm{P} \overline{1}$ & $\mathrm{C}_{62} \mathrm{H}_{40} \mathrm{Cl}_{6} \mathrm{Dy}_{2} \mathrm{~N}_{4} \mathrm{O}_{12}$ & Li et al. (2007b) \\
\hline VICVUZ & $\mathrm{P} \overline{1}$ & $\mathrm{C}_{16} \mathrm{H}_{21} \mathrm{ClCuN}_{3} \mathrm{O} \cdot \mathrm{ClO}_{4}$ & Mikata et al. (2007) \\
\hline WEWZII & $\mathrm{P} \overline{1}$ & $\mathrm{C}_{34} \mathrm{H}_{28} \mathrm{O}_{14} \cdot \mathrm{C}_{3} \mathrm{H}_{6} \mathrm{O} \cdot \mathrm{CHCl}_{3}$ & Tatsuta et al. (2007) \\
\hline WEZBAF & $\mathrm{C} 2$ & $\mathrm{C}_{14} \mathrm{H}_{21} \mathrm{NO}_{7}$ & Metta-Magana et al. (2007) \\
\hline XIJPUC & $\mathrm{P} \overline{1}$ & $\mathrm{C}_{45} \mathrm{H}_{31} \mathrm{~F}_{8} \mathrm{~N}_{2} \mathrm{NiO}_{2} \cdot \mathrm{C}_{24} \mathrm{BF}_{20} \cdot \mathrm{CH}_{2} \mathrm{Cl}_{2}$ & Meinhard et al. (2007) \\
\hline XIKCOK & $\mathrm{P} \overline{1}$ & $\mathrm{C}_{17} \mathrm{H}_{23} \mathrm{Cl}_{4} \mathrm{IrN}_{2} \cdot \mathrm{PF}_{6} \cdot 0.5\left(\mathrm{CH}_{4} \mathrm{O}\right)$ & Corberan et al. (2007) \\
\hline
\end{tabular}


TABLE 2S. Space group revisions; original space group, P1 (No. 1) - Continued

$\begin{array}{lll}\text { Refcode } & \mathrm{S} . \mathrm{G} . & \text { Formula Unit } \\ \text { BOHCOR } & \mathrm{P} \overline{1} & \mathrm{C}_{21} \mathrm{H}_{26} \mathrm{~N}_{2} \\ \text { CIZBOD } & \mathrm{P} \overline{1} & \mathrm{C}_{19} \mathrm{H}_{18} \mathrm{~N}_{2} \mathrm{O}_{2} \mathrm{~S} \\ \text { CONKAS } & \mathrm{P} \overline{1} & \mathrm{C}_{74} \mathrm{H}_{94} \mathrm{~N}_{6} \mathrm{O}_{12} \cdot 2\left(\mathrm{CHCl}_{3}\right) \\ \text { DISHIX } & \mathrm{P} \overline{1} & \mathrm{C}_{18} \mathrm{H}_{20} \mathrm{O}_{4} \\ \text { DISHOD } & \mathrm{P} \overline{1} & \mathrm{C}_{18} \mathrm{H}_{20} \mathrm{O}_{4} \\ \text { DOCFOR } & \mathrm{P} \overline{1} & \mathrm{C}_{30} \mathrm{H}_{30} \mathrm{~N}_{10} \mathrm{Ni}_{2} \mathrm{O}_{6} \\ \text { DOCFUX } & \mathrm{P} \overline{1} & \mathrm{C}_{15} \mathrm{H}_{15} \mathrm{~N}_{5} \mathrm{O}_{3} \mathrm{Zn} \\ \text { DOCXID } & \mathrm{P} \overline{1} & \mathrm{C}_{62} \mathrm{H}_{40} \mathrm{Cl}_{6} \mathrm{~N}_{4} \mathrm{O}_{12} \mathrm{~Tb}_{2} \\ \text { KIYRAM } & \mathrm{C} 2 / \mathrm{c} & \mathrm{C}_{22} \mathrm{H}_{20} \mathrm{~N}_{8} \mathrm{O}_{4} \\ \text { KOMYAN } & \mathrm{P} \overline{1} & \mathrm{C}_{24} \mathrm{H}_{16} \mathrm{Cu}_{2} \mathrm{~N}_{4} \mathrm{O}_{8} \cdot 4\left(\mathrm{H}_{2} \mathrm{O}\right) \\ \text { NOMXUJ } & \mathrm{P} \overline{1} & \mathrm{C}_{36} \mathrm{H}_{48} \mathrm{~N}_{6} \mathrm{Zn} \\ \text { PIXMOZ } & \mathrm{P} \overline{1} & \mathrm{C}_{6} \mathrm{H}_{10} \mathrm{~N}_{2} \cdot 2\left(\mathrm{ClO}_{4}\right) \\ \text { POCMUQ } & \mathrm{P} \overline{1} & \mathrm{C}_{76} \mathrm{H}_{48} \mathrm{~F}_{12} \mathrm{~N}_{4} \mathrm{O}_{8} \mathrm{~Pb}_{2} \\ \text { POKLEH } & \mathrm{Cc} & \mathrm{C}_{21} \mathrm{H}_{25} \mathrm{MnN}_{5} \mathrm{O}_{2}\left(\mathrm{C}_{24} \mathrm{H}_{20} \mathrm{~B}\right) \\ \text { QOFTAH } & \mathrm{P} \overline{1} & \mathrm{C}_{26} \mathrm{H}_{46} \mathrm{~F}_{2} \mathrm{~N}_{2} \mathrm{O}_{2} \mathrm{Sn}_{2} \cdot 2\left(\mathrm{CF}_{3} \mathrm{O}_{3} \mathrm{~S}\right) \\ \text { QQQWPO02 } \mathrm{P} \overline{1} & \mathrm{C}_{10} \mathrm{H}_{8} \mathrm{CuF}_{6} \mathrm{O}_{4} \\ \text { ROJTIA } & \mathrm{P} \overline{1} & \left.\mathrm{C}_{54} \mathrm{H}_{50} \mathrm{Eu}_{2}\right)_{22} \\ \text { YOKPUK } & \mathrm{P} \overline{1} & \mathrm{C}_{24} \mathrm{H}_{36} \mathrm{Ag}_{2} \mathrm{Cl}_{2} \mathrm{~N}_{12} \mathrm{O}_{8} \\ \text { YOMKER } & \mathrm{P} 2{ }_{1} & \mathrm{C}_{12} \mathrm{H}_{13} \mathrm{NO}_{2} \\ \text { XIXCOX } & \mathrm{P} \overline{1} & \mathrm{C}_{128} \mathrm{H}_{146} \mathrm{~N}_{14} \mathrm{Zn}_{2} \cdot 4\left(\mathrm{C}_{6} \mathrm{H}_{6}\right) \cdot 8\left(\mathrm{C}_{2} \mathrm{H}_{3} \mathrm{~N}\right)\end{array}$

Reference

Iglesias et al. (2008)

Cho \& Chang (2008)

Lin et al. (2008)

Cacciapaglia et al. (2008)

Cacciapaglia et al. (2008)

Xing et al. (2008)

Xing et al. (2008)

Li et al. (2008)

Shawali et al. (2008)

Csonka et al. (2008)

Zheng et al. (2008)

Kapoor et al. (2008)

Marandi et al. (2008)

Hureau et al. (2008)

Svec et al. (2008)

Baidina et al. (2008)

Yan et al. (2008)

Luo et al. (2008)

Chmielewski et al. (2008)

Maeda et al. (2008) 


\section{References}

Adams, H., Fenton, D. E., \& McHugh, P. E. (2004). Inorg. Chem. Commun. 7, 147.

Anand, V. G., Saito, S., Shimizu, S. \& Osuka, A. (2005). Angew. Chem. Int. Ed. 44, 7244 .

Ananikov, V. P., Malyshev, D. A., Beletskaya, I. P., Aleksandrov, G. G., \& Eremenko, I. L. (2005). Adv. Synth. Catal. 347, 1993.

Babkov, L. M., Baran, Ya., Davydova, N. A., Petrashko, A., \& Unspenskiy, K. E. (2006). Zh. Strukt. Khim. (Russ.) 47, 759.

Baidina. I. A., Stabnikov, P. A., Gromilov, S. A. \& Smolentsev, A. I. (2008). Zh. Strukt. Khim. 49., 989.

Beckett, M. A., Bland, C. C., Horton, P. N., Hursthouse, M. B., \& Varma, K. S. (2007). J. Organomet. Chem. 692, 2832.

Beddie, C., Hollink, E., Wei, P., Gauld, J. \& Stephan, D. W. (2004). Organometallics 23, 5240 .

Boyle, T. J., Sewell, R. M., Ottley, L. A. M., Pratt III, H. D., Quintana, C. J., \& Bunge, S. D. (2007). Inorg. Chem. 46, 1825.

Bradley, C. A., Flores-Torres, S., Lobkovsky, E., Abruna, H. D. \& Chirik, P. J. (2004). Organometallics 23, 5332 .

Brady, C., Callaghan, P. L., Ciunik, Z., Coates, C. G., Dossing, A., Hazell, A., McGarvey, J. J., Schenker, S., Toftlund, H., Trautwein, A. X., Winkler, H., \& Wolny, J. A. (2004). Inorg. Chem. 43, 4289

Cacciapaglia, R., Di Stefano, S., Mandolini, L., Mencarelli, P., \& Ugozzoli, F. (2008). Eur. J. Org. Chem. 186.

Carver, C. T., Monreal, M. J., \& Diaconescu, P. L. (2008). Organometallics 27, 363.

Champouret, Y. D. M., Marechal, J.-D., Dadhiwala, I., Fawcett, J., Palmer, D., Singh, K. \& Solan, G. A. (2006). Dalton Trans. 2350.

Chantarasiri, N., Ruangpornvisuti, V., Muangsin, N., Detsen, H., Mananunsap, T., Batiya, C., \& Chaichit, N. (2004). J. Mol. Struct. 701, 93.

Chawla, H. M., Singh, S. P., \& Upreti, S. (2007).Tetrahedron 63, 5636. 
Chen, H.-C., Hu, H.-L., Chan, Z.-K., Yeh, C.-W., Jia, H.-W., Wu, C.-P., Chen, J.-D., \& Wang, J.-C. (2007). Cryst. Growth Des. 7, 698.

Cheng, Y. \& Cheng, L.-Q. (2007). J. Org. Chem. 72, 2625.

Chmielewski, M., Cierpucha, M., Kowalska, P., Kwit, M. \& Frelet, J. (2008). Chirality 20, 621 .

Cho, S. H. \& Chang, S. (2008). Angew. Chem. Int. Ed. 47, 2836.

Comelles, J., Pericas, A., Moreno-Maas, M., Vallribera, A., Drudis-Sol, G., Lledos, A., Parella, T., Roglans, A., Garcia-Granda, S., \& Roces-Fernandez, L. (2007). J. Org. Chem. 72, 2077.

Corberan, R., Sanau, M., \& Peris, E. (2007). Organometallics 26, 3492.

Costa, M., Ca, N. D., Gabriele, B., Massera, C., Salerno, G. \& Soliani, M. (2004). J. Org. Chem. 69, 2469.

Csonka, R., Kaizer, J., Giorgi, M., Reglier, M., Hajba, L., Mink, J., \& Speier, S. (2008). Inorg. Chem. 47., 6121.

Diez-Gonzales, S., Stevens, E. D., Scott, N. M., Petersen, J. L., \& Nolan, S. P. (2008). Chem.-Eur. J. 158.

Dodds, C. A., Reglinski, J., \& Spicer, M. D. (2006). Chem.-Eur. J. 12, 931.

Du, J. -Y. (2004). Transition Met. Chem. 29, 699.

Fernandez-Fernandez, M. del C., Bastida, R., Macias, A., Valencia, L., \& PerezLourido, P. (2006a). Polyhedron 25, 783.

Fernandez-Fernandez, M. del C., Bastida, R., Macias, A., Perez-Lourido, P. \& Valencia, L. (2006b). Inorg. Chem. 45, 2266.

Fructos, M. R., Belderrain, T. R., de Fremont, P., Scott, N. M., Nolan, S. P., DiazRequejo, M. M., \& Perez, P. J. (2005). Angew. Chem., Int. Ed. 44, 5284.

Gaspar, A. B., Agusti, G., Martinez, V., Munoz, M. C., Levchenko, G., \& Real, J. A. (2005). Inorg. Chem. Acta 358, 4089.

Gholivand, Kh., Vedova, C. O. D., Firooz, A. A., Alizadehgan, A. M., Michelini, M. C. \& Diez, R. P. (2005). J. Mol. Struct. 750, 64.

Golovko, V. B., Hope-Weeks, L. J., Mays, M. J., McPartlin, M., Sloan, A. M. \& Woods, A. D. (2004). New J. Chem. (Nouv. J. Chim.) 28, 527. 
Hanessian, S., Yun, H., Hou, Y. \& Tintelnot-Blomley, M. (2005). J. Org. Chem. 70, 6746.

Hasegawa, A., Naganawa, Y., Fushimi, M., Ishihara, K., \& Yamamoto, H. (2006). Organic Letters 8, 3175.

Hatano, M., Asai, T., \& Ishihara, K. (2006). Chem. Lett. 35, 172.

Heppeler, A., Andre, J. P., Baschmann, I., Wang, X., Reubi, J.-C., Hennig, M., Kaden, T. A. \& Maeke, H. R. (2008). Chem.-Eur. J. 14, 3026.

Hintermann, L., Lang, F., Maire, P., \& Togni, A. (2006). Eur. J. Inorg. Chem. 1397.

Hureau, C., Groni, S., Guillot, R., Blondin, C., Duboc, C. \& Anxolabehere-Mallart, E. (2008). Inorg. Chem. 47, 9238.

Ie, Y., Nitani, M., \& Aso, Y. (2007). Chem. Let. 36, 1326.

Iglesias, M., Beetstra, D. J., Knight, J. C., Ooi, L.-L., Stasch, A., Coles, S., Male, L., Hursthouse, M. B., Cavell, K. J., Dervisi, A. \& Fallis, I. A. (2008). Organometallics 27, 3279 .

Imamoto, T., Nishimura, M., Koide, A., \& Yoshida, K. (2007). J. Org. Chem. 72, 7413.

Jitzsukawa, K., Oka, Y., Yamaguchi, S. \& Masuda, H. (2004). Inorg. Chem. 43, 8119 .

Kang, J.-G., Kim, T.-J., Kang, H.-J., \& Kang, S. K. (2005). J. Photochem. Photobiol. A: Chem. 174, 28.

Kapoor, I. P. S., Srivastava, P., Singh, G., Singh, U. P., \& Frohlich, R. (2008). J. Phys. Chem. A112, 652.

Kavala, V., Murru, S., Patel, B. K., \& Das, G. (2007). J. Chem. Cryst. 37, 527.

Lan, C.-L., Zhang, S.-H., \& Jiang, Y.-M. (2005). Wuji Huaxue Xuebao (Chin. J. Inorg. Chem.) 21, 1122.

Li, X., Zhang, T.-T., Ju, Y.-L., Wang, C.-Y., Li, Y.-Q., Zhang, L., \& Zhang, Q. (2007b). J. Coord. Chem. 60, 2121.

Li, X., Ju, Y.-L., \& Li, Y.-Q. (2008). J. Coord. Chem. 61, 692.

Li, Y., Chan, P. W. H., Zhu, N.-Y., Che, C.-M., \& Kwong, H.-L. (2004). Organometallics 23, 54 . 
Li, Y.-Q., Ju, Y.-L., Zhang, Y.-B., Wang, C.-Y., Zhang, T.-T., \& Li, X. (2007a). Wuji Huaxue Xuebao (Chin. J. Inorg. Chem.) 23, 969.

Liang, H.-C., Zhang, Y., \& Hetu, M. M. (2007). Inorg. Chem. Comm. 10, 204.

Lin, J.-B., Xu, X.-N., Jiang, X.-K., \& Li, Z.-T. (2008). J. Org. Chem. 73, 9403.

Lin, Q., Wei, T.-B., \& Zhang, Y.-M. (2007). Phosphorus, Sulfur, Silicon, Related Elem. 182, 863 .

Liskin, D. V. \& Valente, E. J. (2008). J. Mol. Struct. 878, 149.

Lorenz, V., Giessmann, S., Gun'ko, Y. K., Fischer, A. K., Gilje, J. W., \& Edelmann, F. T. (2004). Angew. Chem., Int. Ed. 43, 4603.

Lundberg, D., Ullstrom, A.-S., D'Angelo, P., Warminska, D., \& Persson, I. (2007). Inorg. Chem. Acta 360, 2744.

Luo, G.-G., Huang, R.-B., Chen, J.-H., Lin,, L.-P. \& Zheng, L.-S. (2008). Polyhedron 27, 2791.

Ma, C., Han, Y., \& Zhang, R. (2004a). J. Organomet. Chem. 689, 1675.

Ma, C., Zhang, J. \& Zhang, R. (2004b). Heteroat. Chem. 15, 338.

Ma, C., Li, J., Zhang, R., \& Wang, D. (2006). Inorg. Chim. Acta 359, 2407.

Maeda, C., Yamaguchi, S., Ikeda, C., Shinokubo, H., \& Osuka, A. (2008). Organic Letters 10, 549 .

Malyshev, D. A., Scott, N. M., Marion, N., Stevens, E. D., Ananikov, V. P., Beletskaya, I. P., \& Nolan, S. P. (2006). Organometallics 25, 4462.

Marandi, F., Asghari-Lalami, N., Ghorbanloo, M., \& Mcardle, P. (2008). J. Coord. Chem. 61, 1545.

Mas-Marza, E., Poyatos, M., Sanau, M., \& Peris, E. (2004). Inorg. Chem. 43, 2213.

Mayoral, M. J., Cano, M., Campo, J. A., Heras, J. V., Pinilla, E., \& Torres, M. R. (2004). Inorg. Chem. Commun. 7, 974.

Meinhard, D., Wegner, M., Kipiani, G., Hearley, A., Reuter, P., Fischer, S., Marti, O., \& Rieger, B. (2007). J. Amer. Chem. Soc. 129, 9182.

Metta-Magana, A. J., Reyes-Martinez, R., \& Tlahuext, H. (2007). Carbohydrate Research 342, 243. 
Mikata, Y., Fujimoto. T., Sugai, Y., \& Yano, S. (2007). Eur. J. Inorg. Chem. 1143.

Mizobe, Y., Miyata, M., Hisaki, I., \& Tohnai, N. (2007). Chem. Let. 36, 280.

Montiel-Palma, V., Huitron-Rattinger, E., Cortes-Llamas, S., Munoz-Hernandez, M.A., Garcia-Montalvo, V., Lopez-Honorato, E., \& Silvestru, C. (2004). Eur. J. Inorg. Chem., 3743.

Morita, T., Asada, Y., Okuda, T., \& Nakashima, S. (2006). Bull. Chem. Soc. Jpn. 79, 738 .

Morozova, N. B., Stabnikov, P. A., Baidina, I. A., Semyannikov, P. P., Trubin, S. V., \& Igumenov, I. K. (2007). Zh. Strukt. Khim. (Russ.) 48, 947.

Muller, T., Seichter, W., \& Weber, E. (2006). New J. Chem. 30, 751.

Muthusamy, S., Krishnamurthi, J. \& Nethaji, M. (2005). Chem. Comm. 3862.

Ng, M. T., Deivaraj, T. C., Klooster, W. T., McIntyre, G. J., \& Vittal, J. J. (2004). Chem. - Eur. J. 10, 5853.

Ogura, K., Ooshima, K., Akazome, M., \& Matsumoto, S. (2006). Tetrahedron 62, 2484.

Oliva, A. I., Simon, L., Muniz, F. M., Sanz, F., Ruiz-Valero, C. \& Moran, J. R. (2004). J. Org. Chem. 69, 6883.

Onozaki, K., \& Miyamura, K. (2006). Bull. Chem. Soc. Jpn. 79, 876.

Oyaizu, K., Ohtani, Y., Shiozawa, A., Sugawara, K., Saito, T., \& Yuasa, M. (2005). Inorg. Chem. 44, 6915.

Pellicani, R. Z., Intini, F. P., Maresca, L., Mesto, E., Pacifico, C., \& Natile, G. (2006). Eur. J. Inorg. Chem. 1635.

Plante, J. B. \& Glass, T. E. (2006). Organic Letters 8, 2163.

RoyChowdhury, S., Komiyama, T., Yukawa, Y., \& Bhattacharyya, R. (2004). Inorg. Chem. Commun. 7, 1117.

Sakakura, A., Kondo, R., Umemura, S., \& Ishihara, K. (2007). Adv. Synth. Catal. 349, 1641.

Sarkar, B., Bocelli, G., Cantoni, A., \& Ghosh, A. (2008). Polyhedron 27, 693. 
Shawali, A. S., Mosselhi, M. A. N., Abdallah, M. A. \& Elewa, M. S. (2008). J. Chem. Res., 67.

Shen, Y.-M., Duan, W.-L., \& Shi, M. (2004). Eur. J. Org. Chem. 3080.

Shi, J.-M., Sun, Y.-M., Liu, Z., \& Liu, L.-D. (2006). Chem. Phys. Lett. 418, 84.

Shi, J., Xu, J.-N., Song, T.-Y., He, X., Ye, J.-W., Wang, L., Fan, Y., \& Zhang, P. (2007). J. Coord. Chem. 60, 295.

Shi, X., Zhu, G., Wang, X., Li, G., Fang, Q., Wu, G., Tian, G., Xue, M., Zhao, X., Wang, R. \& Qiu, S. (2005). Crystal Growth Des. 5, 207.

Shiga, T., Okawa, H., Kitagawa, S., \& Ohba, M. (2006). J. Am. Chem. Soc. 128, 16426.

Shimasaki, T., Kato, S., Ideta, K., Goto, K., \& Shinmyozu, T. (2007). J. Org. Chem. 72, 1073.

Song, L.-C., Cheng, H.-W., \& Hu, Q.-M. (2004). J. Organomet. Chem. 689, 1849.

Song, L.-C., Gao, J., Wang, H.-T., Hua, Y.-J., Fan, H.-T., Zhang, X.-G., \& Hu, Q.-M. (2006). Organometallics 25, 5724.

Suen, M.-C., Wang, Y.-H., Hsu, Y.-F., Yeh, C.-W., Chen, J.-D., \& Wang, J.-C. (2005). Polyhedron 24, 2913

Svec, P., Padelkova, Z., Cerbosek, Z., De Proft, F. \& Ruziki, A. (2008). J. Organomet. Chem. 693, 2937.

Tao, R.-J., Mei, C.-Z., Liu, B.-T. \& Niu, J.-Y. (2006). Chin. J. Chem. 24, 1559.

Tatsuta, K., Kasai, S., Amano, Y., Yamaguchi, T., Seki, M., \& Hosokawa, S. (2007). Chem. Lett. 36, 10.

Tian, C.-H., Sun, Z.-G., Li, J., Zheng, X.-F., Liang, H.-D., Zhang, L.-C., You, W.-S., \& Zhu, Z.-M. (2007a). Inorg. Chem. Commun. 10, 757.

Tian, L., Yu, L.-C., Chen, L., \& Xia, W.-Q. (2007b). J. Coord. Chem. 60, 1847.

Ullstrom, A.-S., Warminska, D. \& Persson, I. (2005). J. Coord. Chem. 58, 611

Uncuta, C., Bartha, E., Tanase, C. I., Tanase, A. E., Costan, O., Ciuca, M., Vanthuyne, N., \& Roussel, C. (2006). ARKIVOC 7, 42-10. 
Voskressensky, L. G., Borisova, T. N., Kostenev, I. S., Vorobiev, I. V., \& Varlamov, A. V. (2005). Tetrahedron Letters 46, 1975.

Wang, M.-A., Tu, G.-Z., Ma, Z.-C., Zhang, N., \& Wang, D.-Q. (2006). Chin. J. Chem. 24, 205.

Wang, X.-L., Chen, B.-K., Lin, H.-Y., Bi, Y.-F. \& Liu. G.-C. (2008). J. Chem. Cryst. $\mathbf{3 8}, 339$.

Wang, Y., Huang, S., \& Xia, P. (2005). Synth. Commun. 35, 3141.

Xing, Y. H., Han, J., Zhou, G. H., Sun, Z., Zhang, X. J., Zhang, B. L., Zhang, Y. H., Yuan, H. Q. \& Ge, M. F. (2008) J. Coord. Chem. 61, 715.

Yamada, H., Okajima, K., Imagawa, H., Mukae, T., Kawamura, Y., \& Nishizawa, M. (2007). Bull. Chem. Soc. Jpn, 80, 583.

Yan, J., Guo, Y., Li, H., Sun, X. \& Wang, Z. (2008). J. Mol. Struct. 891, 298.

Yang, L., Su, Y., Xu, Y., Zhang, S., Wu, J. \& Zhao, K. (2004). J. Inorg. Biochem. 98, 1251.

Yeh, C.-W., Yen, Y.-S., Lin, W.-B., Su, C.-W., Chen, J.-D. \& Wang, J.-C. (2005). Struct. Chem. 16, 141.

Yoshikawa, N., Yamabe, S., Kanehisa, N., Kai, Y., Takashima, H., \& Tsukahara, K. (2007). Eur. J. Inorg. Chem. 1911.

Yu, G.-A., Ren, Y., Guan, J.-T., Lin, Y., \& Liu, S. H. (2007). J. Organomet. Chem. 692, 3914 .

Yu, J.-H., Wei, Y.-G., Ding, C.-J., Du, H.-G., Yan, S.-G., \& Guo, H.-Y. (2006). Chin. J. Chem. 24, 695 .

Zhang, A., Ferguson, J. S., Yamato, K., Zheng, C. \& Gong, B. (2006). Organic Letters. 8, 5117.

Zharkova, G. I., Baidina, I. A., Stabnikov, P. A. \& Igumenov, I. K. (2006). Zh. Strukt. Khim. (Russ.) 47, 731.

Zheng, L., Yang, F., Dang, Q. \& Bai, X. (2008). Org. Let. 10, 889.

Zheng, Z., Elmkadden, M. K., Fischmeister, C., Roisnel, T., Thomas, C. M., Carpentier, J.-F., \& Renuad, J.-L. (2008). New J. Chem., 2150. 
Zherikova, K. V., Morozova, N. B., Peresypkina, E. V., Baidina, I. A., \& Igumenov, I. K. (2006). Zh. Strukt, Khim. (Russ.) 47, 581.

Zhu, H.-F., Fan, J., Okamura, T., Sun, W.-Y. \& Ueyama, N. (2005). Cryst. Growth Des. 5, 289.

Zhu, N., Lightsey, D., Foroozesh, M., Alworth, W., Chaudhary, A., Willett, K. L. \& Stevens, C. L. K. (2006). J. Chem. Cryst. 36, 289.

Zhu, Q., Chu, W., Huang, R., Zhang, J., \& Xu, Y. (2008). J. Coord. Chem. 61, 3390.

Zinn, P. J., Sorrell, T. N., Powell, D. R., Day, V. W. \& Borovik, A. S. (2007). Inorg. Chem. 46, 10120. 